\title{
Three Pipe Corrosion Failure Cases
}

\author{
Andrew Havics
}

pH2, LLC, Avon, IN

Pipe fails for a number of reasons, but often corrosion, and it corrodes because of a variety of causes; dissimilar metals, microbially induced corrosion (MIC), product incompatibility, erosion corrosion, chemical treatment or lack thereof, temperature induced diffusion or recrystallization, and others are the potential culprits. Two water-based systems and one glycol system were examined metallurgically and biologically to ascertain the most likely causes of the corrosion and their relation to failure.

Examinations typically start with macro-observations using stereo or dissecting scopes, followed by subsample material preparation, then light microscopy including reflected light with polarized light, Normarski DIC, metallographic etching, hardness testing, and scanning electron microscopy with energy dispersive x-ray analysis (SEM-EDS). In two of these cases, filtered water from the affected and nonaffected areas were also examined for both biological and non-biological components using microscopical techniques, such as staining and epi-fluorescence, micro-chemical and SEM-EDS, which can help point to a cause, or lack of one. These techniques are supplemented by other analyses such as micro-hardness, wet chemistry (dissolved oxygen, $\mathrm{pH}$, alkalinity), corrosion testing, etc.

In Case 1, a glycol-based cooling system leaked. Examination of the pipe revealed extensive corrosion on both the exterior (very unusual) and interior of the piping. The microstucture was consistent and there no signs of microbially induced corrosion (MIC). A review of electrochemical potential testing revealed the most likely cause was galvanic action with the fluid.

Case 2 involved a pipe utilized in condenser water supply and return in an open system on ocean front equivalent property. The leaks were reported to have developed in the longitudinal welds. Light and electron microscopy revealed a flaking morphology, filamentous iron and whisker-like particles of iron, significant tuberculation formation, selective loss of material at the weld, slight change in microhardness at the weld seam (with no apparent bulk compositional change), preferential etching at the weld seam. Water testing data indicated a fluid with corrosive significant potential. Results support weld seam failure from corrosion (as opposed to poor weld construction), and most likely a galvanic corrosion.

Case 3 involved a pipe utilized in an open cooling water supply on a multiple story condo complex. Leaks were reported to have developed in welds. Light and electron microscopy revealed significant corrosion including large tubercles on the interior of pipes of all dimensions (suggesting a common source). Corrosion pitting was less than $150 \mathrm{um}$ in depth, and was not preferential to the weld area. There were some differences in micro-structure (revealed best by via preferential etching) but no correlation to corrosion. Some differences in micro-hardness were most likely attributed to corrosion via correlation with oxygen by depth. There were indications that MIC was involved to a lesser, but still deleterious, degree. This was possibly a secondary aspect of tubercle formation. MIC was not likely Sulfate Reducing Bacteria (SRB) or iron-fixing organisms given the evidence such as negative lead acetate test, low sulfur without enrichment, and no SEM evidence of stalks. MIC would have been influenced by the maintenance and quality of the water in the system. The presence of significant bacteria (active and inactive), diatoms, nematodes, etc. in water samples and diatoms embedded in 
corrosion materials, clearly and strongly implicate poor water quality control. Poor water quality control was secondarily supported by the presence of Chlorine $(\mathrm{Cl})$ in corrosion products evaluated by SEMEDS, generation rate of tubercles, as well as water $\mathrm{pH}$ and conductivity variability. Overall, the corrosion and subsequent pitting were attributed to poor water quality control.

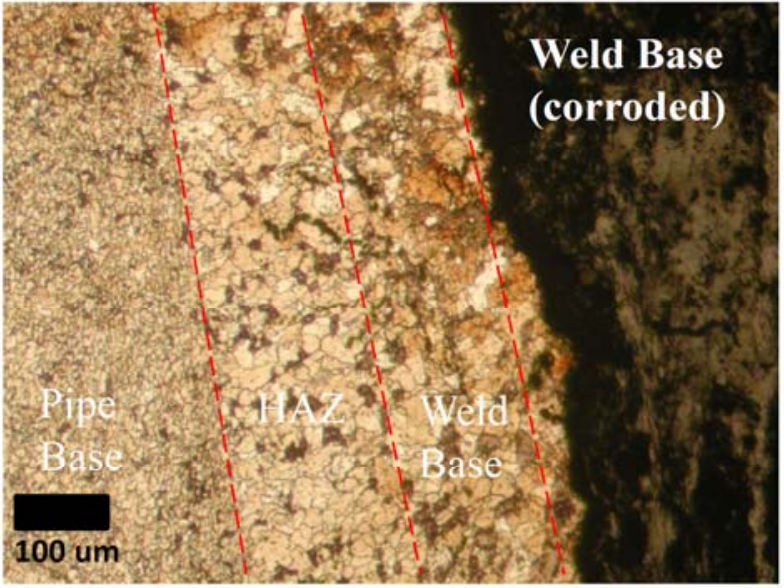

Figure 1. Photomicrograph of etched pipe cross section from Case 1 through left half of the weld. It includes the pipe base metal, heat affected zone (HAZ), weld base metal, and corroded (missing) weld metal.

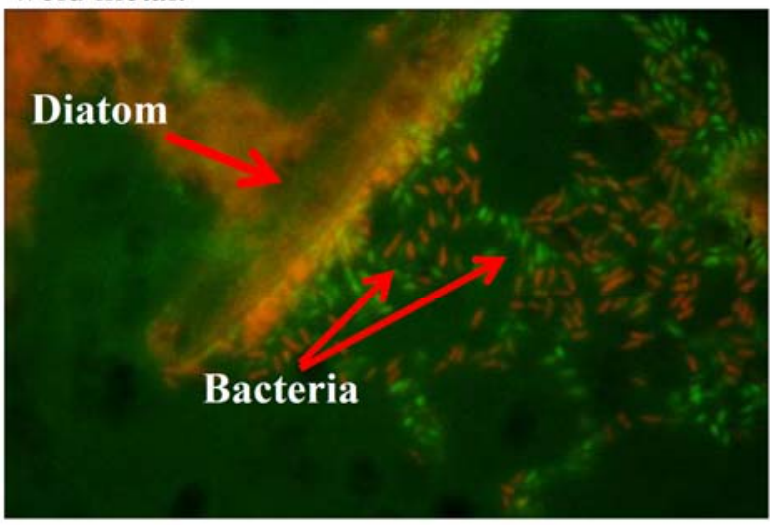

Figure 3. Photomicrograph of filtered water sample from Case 2. Biomaterials (biofilm on a a diatom and bacteria) are stained with Acridine orange $(\mathrm{AO})$ and viewed via epi-fluorescence.

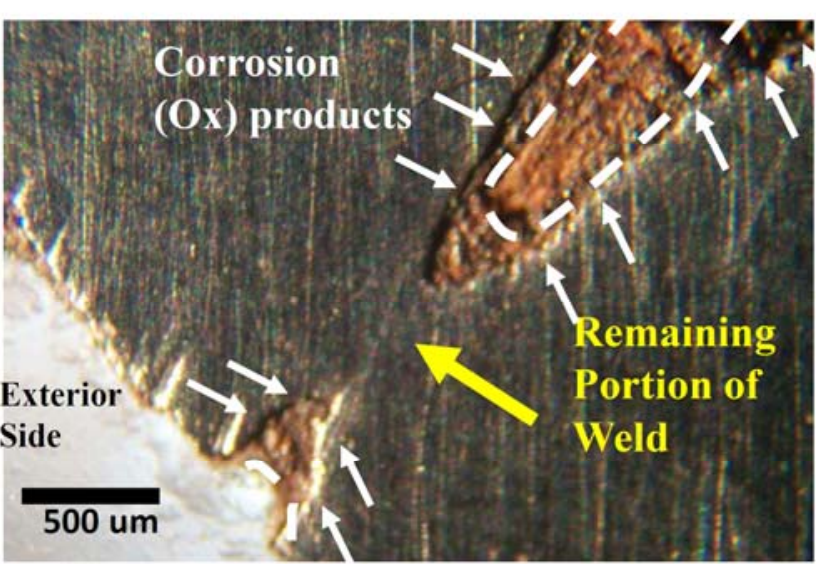

Figure 2. Photomicrograph of a ground but unpolished pipe cross section from Case 1 showing corroded weld on exterior and mid-interior surface.

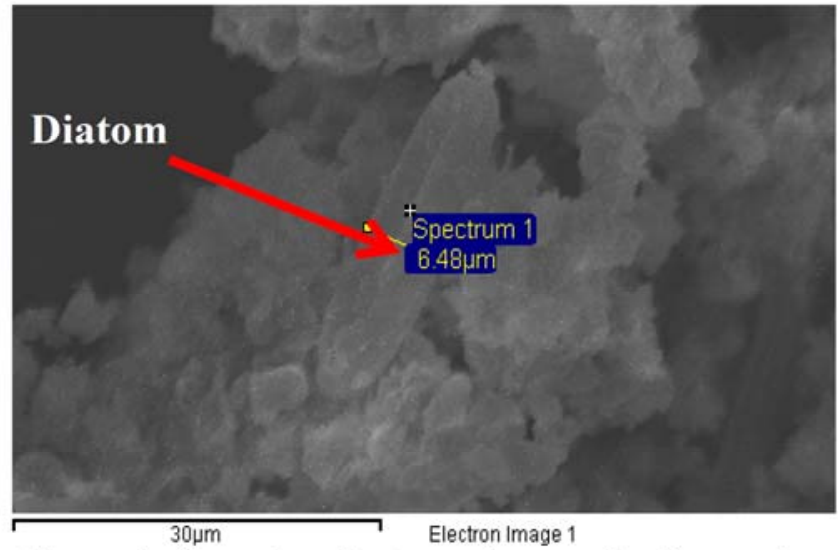

Figure 4. Scanning electron micrograph of corrosion product on interior side of pipe sample with a diatom embedded in the corrosion layer.

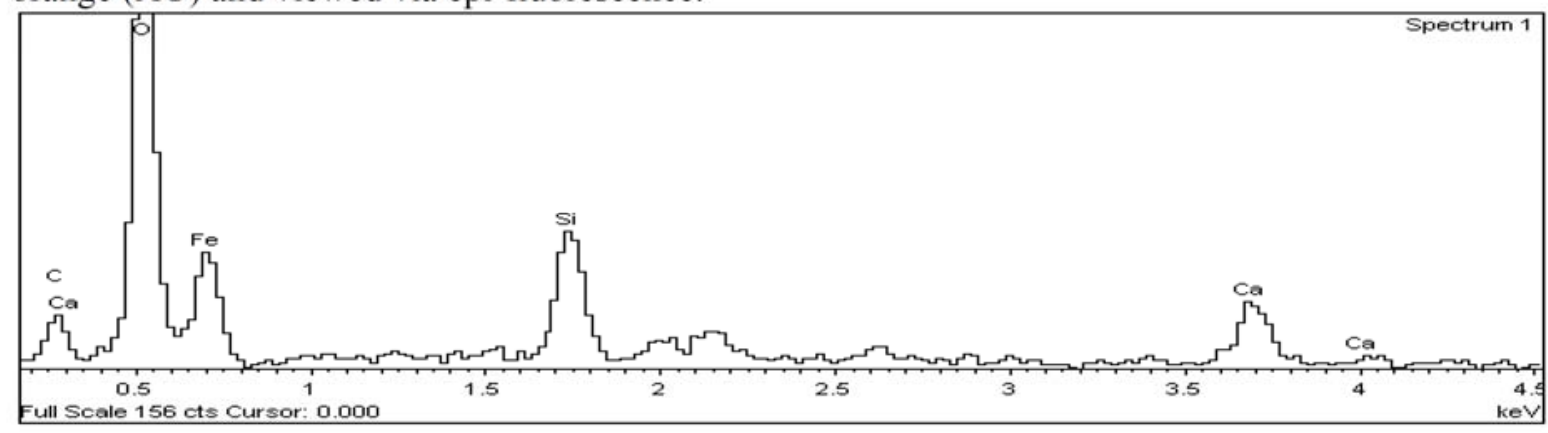

Figure 5. EDS spectra from diatom shown in Figure 4, where the diatom is composed of Si \& O, whereas the $\mathrm{Ca}$ and $\mathrm{Fe}$ are from the water deposits and corroded pipe, respectively. 